\title{
Identification of trunk mutations in gastric carcinoma: a case study
}

\author{
Zhan Zhou ${ }^{1 \dagger}$, Shanshan $\mathrm{Wu}^{1 \dagger}$, Jun Lai ${ }^{1}$, Yuan Shi ${ }^{2}$, Chixiao Qiu' ${ }^{1}$ Zhe Chen ${ }^{2}$, Yufeng Wang ${ }^{3}$, Xun Gu', \\ Jie Zhou ${ }^{1 *}$ and Shuqing Chen ${ }^{1,5^{*}}$
}

\begin{abstract}
Background: Intratumor heterogeneity (ITH) poses an urgent challenge for cancer precision medicine because it can cause drug resistance against cancer target therapy and immunotherapy. The search for trunk mutations that are present in all cancer cells is therefore critical for each patient.

Case presentation: In this study, we aimed to evaluate the efficiency of multiregional sequencing for the identification of trunk mutations present in all regions of a tumor as a case study. We applied multiregional wholeexome sequencing (WES) to investigate the genetic heterogeneity and homogeneity of a case of gastric carcinoma. Approximately $83 \%$ of common missense mutations present in two samples and approximately $89 \%$ of common missense mutations present in three samples were trunk mutations. Notably, trunk mutations appeared to have higher variant allele frequencies (VAFs) than non-trunk mutations.
\end{abstract}

Conclusions: Our results indicate that small-scale multiregional sampling and subsequent screening of low VAF somatic mutations might be a cost-effective strategy for identifying the majority of trunk mutations in gastric carcinoma.

Keywords: Intratumor heterogeneity, Gastric carcinoma, Multiregional sampling, Cancer somatic mutation, Cancer evolution

\section{Background}

Cancer is believed to be driven by somatic mutations, including single nucleotide variations (SNVs), small insertions/deletions (INDELs), copy number variations, structural variations, and epigenetic changes $[1,2]$. In addition to their role in oncogenesis, cancer somatic mutations are potential biomarkers for cancer diagnosis and target therapy $[3,4]$. As long as the somatic mutation results in the production of new epitopes on the membrane of the tumor cell, the active protein arising from the mutation will cause the immune system to recognize the affected cell as foreign. Thus, such proteins are considered ideal targets for cancer immunotherapy, which is now called neoantigen [5]. With the development of cancer genomics, tumor-specific neoantigens have attracted much attention in current biomedical

\footnotetext{
* Correspondence: zhoujie127@zju.edu.cn; chenshuqing@zju.edu.cn

${ }^{\dagger}$ Equal contributors

${ }^{1}$ College of Pharmaceutical Sciences, Zhejiang University, Zhejiang,

Hangzhou 310058, China

Full list of author information is available at the end of the article
}

research because of their potential to be ideal targets for cancer immunotherapy [6-10]. Unfortunately, large-scale cancer genome sequencing analyses have revealed that cancer is a heterogeneous disease and that no two cancers harbor the same complement of somatic mutations. Additionally, there is no common set of mutated genes in all cancers [11-13]. Intertumor heterogeneity suggests that cancer is a personalized disease. Only a very small number of genes (such as TP53) are recurrently mutated in more than $10 \%$ of cancer patients [14]. Therefore, theoretically, somatic mutations should be analyzed for each individual cancer patient to conduct precision diagnosis and precision treatment.

Furthermore, recent studies involving multiregional genome sequencing and single-cell sequencing in different tumor types have revealed that cancer is not only heterogeneous between tumors but also highly heterogeneous within tumors [15-21]. Intratumor heterogeneity (ITH) is a major challenge to cancer precision medicine because it might lead to an underestimation of the cancer somatic mutation landscape based on a single tumor 
biopsy and might contribute to failure in drug treatment and the emergence of drug resistance [22]. Therefore, a single tumor biopsy might be insufficient for identifying all cancer somatic mutations in that tumor because such a biopsy cannot distinguish trunk mutations that are present in all regions of the tumor, branch mutations that are present in only some regions of the tumor, and private branch mutations that are present only in one region of the tumor [15]. Cancer is widely accepted to be a microevolutionary process that originates from a single cell $[2,23]$. Determining the phylogeny of cancer evolution will help identify trunk, branch, and private branch mutations. The identification of trunk mutations is critical for biomarker development and cancer precision medicine, because these mutations represent the genomic differences between all cancer cells and normal cells. Specifically, trunk mutations within protein-coding regions might result in mutant proteins, which are potential tumor-specific neoantigens in all cancer cells.

In this study, multiregional whole-exome sequencing (WES) was applied to identify the intratumor heterogeneity and homogeneity of gastric carcinoma through a case study. We classified these mutations into trunk, branch, and private branch mutations by comparing the somatic nonsynonymous substitutions within six different regions of the primary tumor from a male gastric cancer patient. Private branch mutations show ITH, whereas trunk mutations indicate homogeneity. We further discussed the most cost-effective number of regions required to identify the majority of trunk mutations and compared the mutation frequencies between trunk and non-trunk mutations based on both our case study and published data for multiregional samples of 11 surgically resected lung adenocarcinomas [15].

\section{Case presentation}

The gastric cancer patient was a 61-year-old male who underwent tumor-reductive surgery in July 2015. The postoperative pathological analysis showed that the tumor was a moderately differentiated adenocarcinoma with a bulge in the cardiac gastric gland. The tumor was $3 \times 2.2 \times 1.2 \mathrm{~cm}$ in size (Fig. 1a) and had invaded all layers of the stomach wall and lower esophagus. The lymph nodes exhibited no tumor cells metastasis.

Six multiregional samples were collected in a clockwise pattern from the surgically resected gastric carcinoma within $30 \mathrm{~min}$ after the surgery; the samples were immediately preserved in liquid nitrogen (Fig. 1b). The collection and use of the patient samples were approved by the Zhejiang Hospital of Traditional Chinese Medicine. Written informed consent was obtained from the participant for publication of this case report. We confirmed that all methods were performed in accordance with the relevant guidelines (Approved Guidelines of the Clinical and Laboratory Standards Institute MM01-A3, MM13-A, and MM20-A, and the CARE Guidelines).

\section{Methods \\ Multiregional WES}

DNA was extracted from six samples of one gastric carcinoma tumor. Exomes were captured from $750 \mathrm{ng}$ of genomic DNA per sample using the Agilent SureSelect Human All Exon V5 Kit (Agilent Technologies, Santa Clara, CA, USA) according to the manufacturer's instructions. Paired-end multiplex sequencing was then performed on the Illumina HiSeq X10 sequencing platform. On average, the sequencing depth was $161 \times$ per sample (ranging from $133 \times$ to $216 \times$ with a standard deviation of $\pm 28 \times$ ).

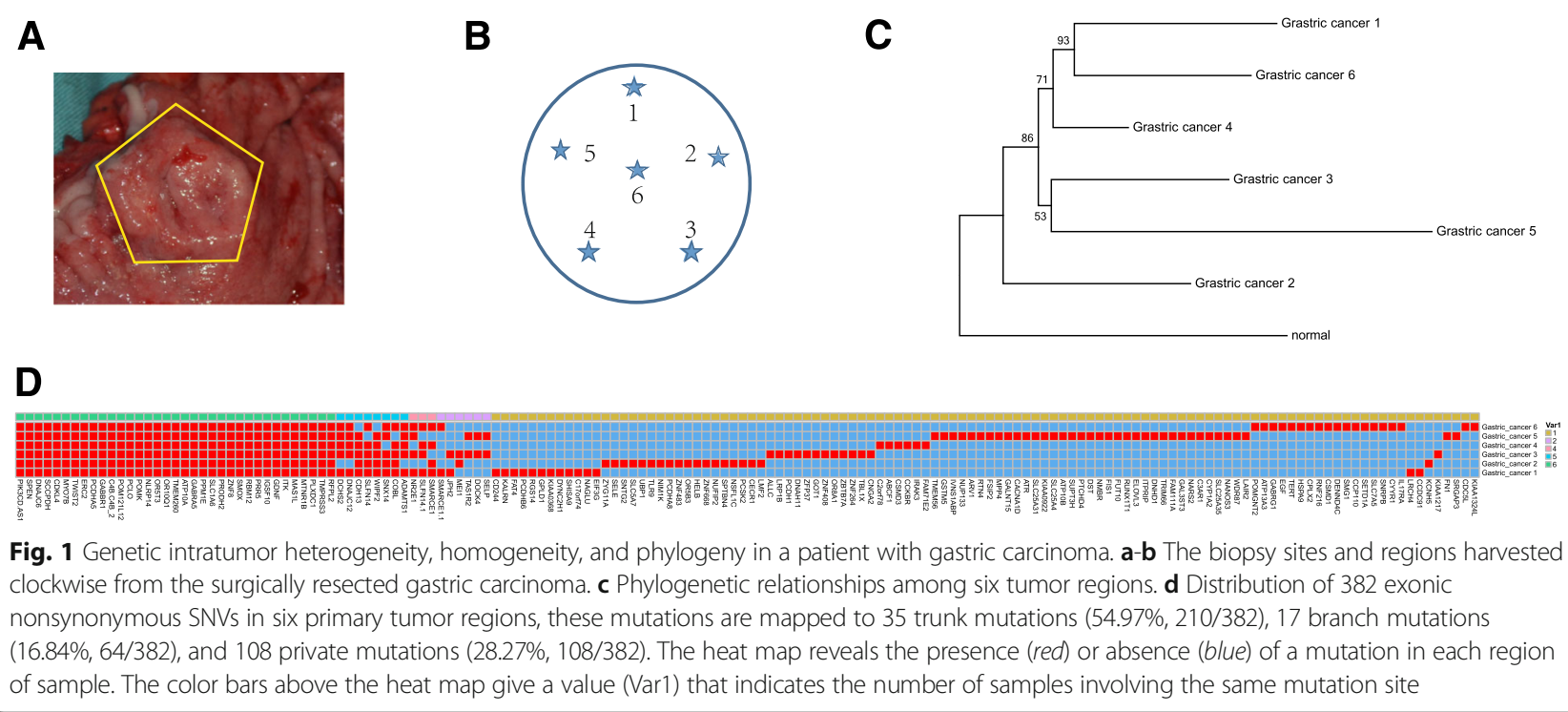




\section{Identification of somatic mutations}

Somatic mutations were identified by the pipeline following the Genome Analysis Toolkit (GATK) best practices for somatic SNV and INDEL discovery in WES [24-26]. Our analysis was mainly focused on exonic mutations, including nonsynonymous SNVs, synonymous SNVs, small INDELs, and stop codon mutations (stopgains). Paired-end reads in the FastQ format were aligned to the reference human genome (GRCh37) using Burrows-Wheeler Aligner (BWAMEM) with the default settings [27]. The aligned reads were further processed by sorting, duplicate removal, INDEL realignment, and base recalibration using Samtools [28], Picard-tools and GATK [24]. Somatic SNVs and small INDELs were detected using MuTect2 [25], which is a built-in package in GATK v3.5. In addition to MuTect2's built-in filters, we applied the following filtering criteria for SNVs: (i) total read count in tumor DNA $\geq 50$; (ii) total read count in germline DNA $\geq 30$; (iii) VAF in tumor DNA $\geq 5 \%$; (iv) VAF in blood DNA $=0$. The following criteria for small INDELs: (i) total read count in tumor DNA $\geq 10$; (ii) total read count in blood DNA $\geq 6$; (iii) VAF in tumor $\geq 5 \%$ and total number of reads supporting a call $\geq 5$; and (iv) VAF in normal DNA $=0$. SNVs and INDELs were annotated using ANNOVAR [29]. Primers were designed to validate the 27 trunk nonsynonymous SNVs shared by the six samples using PCR and sequencing.

Moreover, another set of raw data from the somatic mutations of multiregional samples of 11 surgically resected lung adenocarcinomas [15] was downloaded and analyzed using the same pipeline to investigate the distribution of the mutations across samples. The VAFs of the nonsynonymous SNVs in gastric carcinoma were analyzed based on the relationships between the trunk, branch and private branch mutations, using the Wilcoxon test implemented in R (https://www.rproject.org/).

The UniProtKB/Swiss-Prot (http://www.uniprot.org) and DAVID v6.8 [30] were utilized for functional annotation analysis of mutated genes with trunk, branch and private branch mutations.

\section{Phylogenetic analysis}

The somatic mutation profile for each tumor sample was converted into binary format. All somatic mutations that were present in the exonic regions of at least one tumor sample were used for the phylogenetic analysis. The germline DNA from the blood sample was set as the outgroup with the assumption of somatic mutations. The phylogenetic tree was inferred with the neighborjoining method using MEGA7 [31].

\section{Results}

Identification of somatic mutations by multiregional WES

To evaluate the ITH in gastric carcinoma, multiregional WES was performed on tumor genomic DNA obtained from a patient with gastric carcinoma (Fig. 1a, b); normal genomic DNA was extracted from blood as a reference. WES was conducted at a mean depth of $161 \times$. Across all six regions of the tumor, 1231 simple somatic mutations, including SNVs and small INDELs (Additional file 1), were identified, and these mutations included 539 exonic mutations consisting of nine INDELs, 20 stopgains, 128 synonymous SNVs and 382 nonsynonymous SNVs (Additional file 2: Table S1). The distribution of SNVs in the protein-coding regions, including stopgains as well as synonymous and nonsynonymous SNVs, indicates the phylogenetic relationship across the six samples (Fig. 1c). Most of the somatic mutations in protein-coding regions either occur in only one sample $(28.76 \%, 155 / 539)$ or are common to all samples $(53.42 \%, 288 / 539)$ (Fig. 1d, Additional file 3: Fig. S1 A-B). All 382 of the nonsynonymous SNVs from six samples originated from 160 exact mutation sites (Additional file 4), including 210 nonsynonymous SNVs derived from 35 common mutation sites in all six samples that were classified as trunk mutations $(54.97 \%, 210 / 382)$. Non-trunk mutations did not occur in all of the samples, among these mutations, 108 nonsynonymous SNVs occurred in only one sample and were classified as private branch mutations $(28.27 \%, 108 / 382)$. The other 64 nonsynonymous SNVs were branch mutations that were present in some but not all regions of the tumor.

The 35 trunk mutations occurred on 35 genes, and these genes were called trunk genes; similarly, the private and branch genes were defined as well. For example, SPEN, PRODH2, IGSF10, ITK and MTNR1B were five of the trunk genes, and SMARCE1 was a branch gene harboring mutations from two different sites that was involved in four and two samples. SPEN, PRODH2, IGSF10, MTNR1B and SMARCE1 were found to be mutated in $0.49 \%(1 / 203)$ of esophageal cancers, $0.41 \%(1 / 246)$ of endometrial cancers, $0.52 \%$ (1/194) of cervical cancers, $0.26 \%(1 / 391)$ of pancreatic cancers and $0.55 \%(1 / 183)$ of melanomas, in the International Cancer Genome Consortium (ICGC) database (http://icgc.org/, release 22). An additional eight genes were also found in the ICGC database (Additional file 2: Table S2). Notably, two trunk genes (SPEN and ITK), a branch gene (SMARCE1), and several private genes (FAT4, CACNA1D, ATR, RUNX1T1, TERT, and SRGAP3) were defined as cancer-associated genes, according to the cancer gene census [32,33]. SPEN supports the transcription activation in osteoblasts and is an essential corepressor protein to regulate different key pathways, including the Notch pathway. SPEN can block the precursor $\mathrm{B}$ cells differentiating into marginal zone B 
cells and also repress the transcription via the recruitment of large complexes that contain histone deacetylase proteins [34]. ITK is a tyrosine kinase that plays an essential role in the regulation of the adaptive immune response. ITK also regulates the development, function and differentiation of conventional $\mathrm{T}$ cells and nonconventional NKT cells [35]. SMARCE1 is involved in the transcriptional activation and repression of select genes, and also in the repression of neuronal specific gene promoters in non-neuronal cells through specifically interaction with the CoREST corepressor [36]. Function of the remaining cancer-associated genes and all mutated genes is included in Additional file 2: Table S3 and Additional file 5.

Twenty-seven of 35 trunk mutations were verified by PCR amplification, and 24 trunk mutations were verified in all six tumor samples. However, one mutation in RBM12 was verified in only one sample, and two mutations in SPEN and TWIST2 failed to be verified due to unavailable rational primers. None of the mutations were verified in the blood samples (Additional file 3: Fig. S1C).

\section{Multiregional sequencing can identify the majority of trunk mutations}

In this study, we focused on nonsynonymous SNVs for further analysis because nonsynonymous SNVs could generate tumor-specific mutant proteins. These mutations were shared among the samples (Fig. 2a-c). Almost all of the mutations either occurred in a single sample or were shared by all six samples, in agreement with the heat maps (Fig. 1c, Additional file 3: Fig. S1 A-B). For each sample, trunk mutations made up a large proportion, ranging from $42.68 \%$ to $70.00 \%$, followed by private mutations $(12.00 \%-$ $45.12 \%)$. Private mutations show the degree of ITH, while trunk mutations indicate some homogeneity. We found that approximately $83 \%$ of the common mutations of any two samples and approximately $89 \%$ of the common mutations of any three samples were trunk mutations (Fig. 2d-f), which indicated that sampling of two or three regions could identify the majority of trunk mutations in the gastric carcinoma of the patient.

To explore the effectiveness of small-scale multiregional sampling in the identification of trunk mutations, we conducted a secondary analysis of a published dataset on a different cancer type, which was a multiregional WES of 48 regions from 11 resected lung adenocarcinomas [15]. The exonic nonsynonymous SNVs from the somatic mutations of 48 samples were analyzed in the same way as in the gastric carcinoma study. We found that most mutations across each tumor sample were trunk mutations (Additional file 3: Fig. S2). On average, $86.29 \%$ of the exonic nonsynonymous SNVs were trunk mutations (Fig. 3a). More than $82 \%$ of the shared

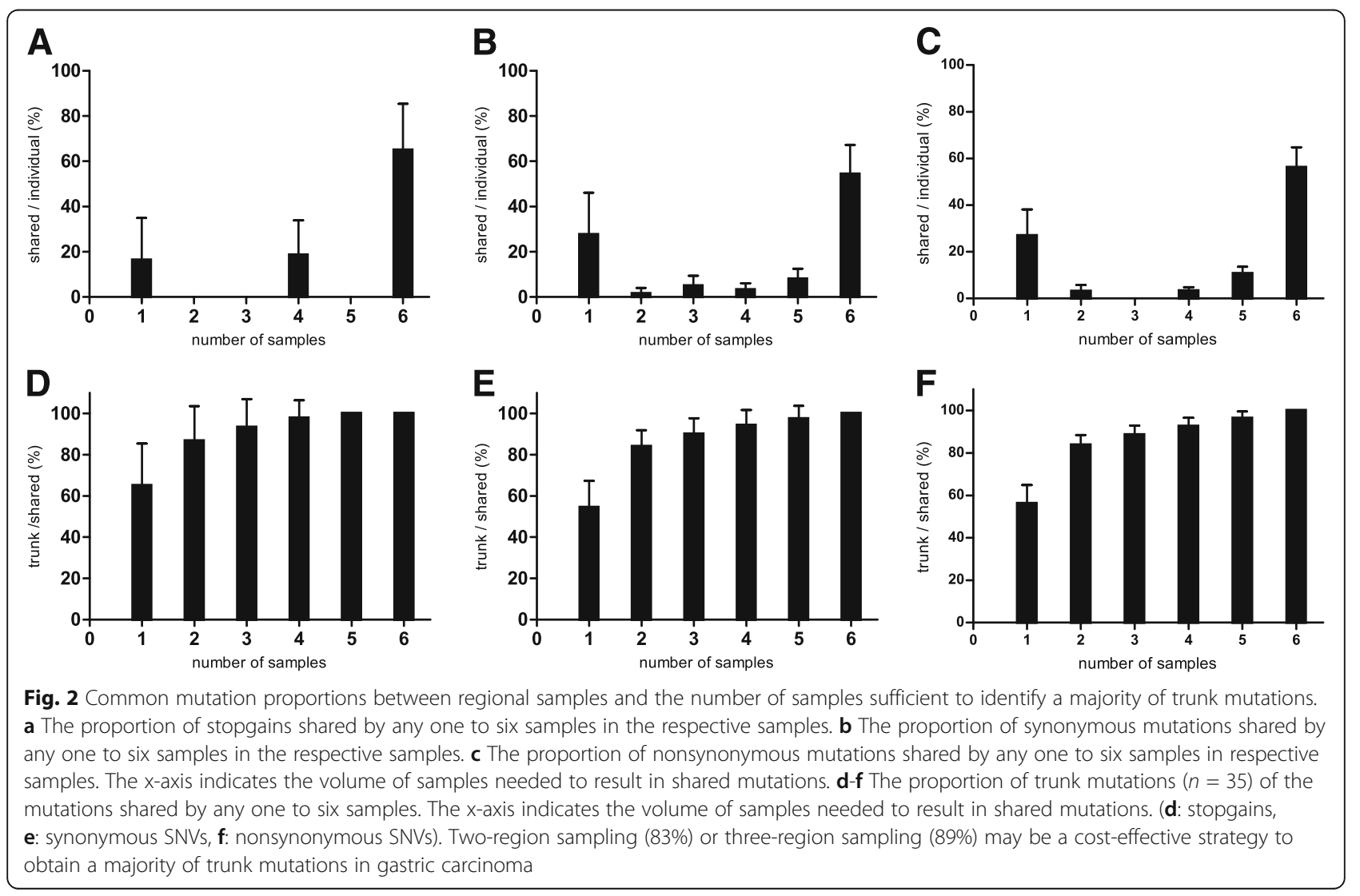


A

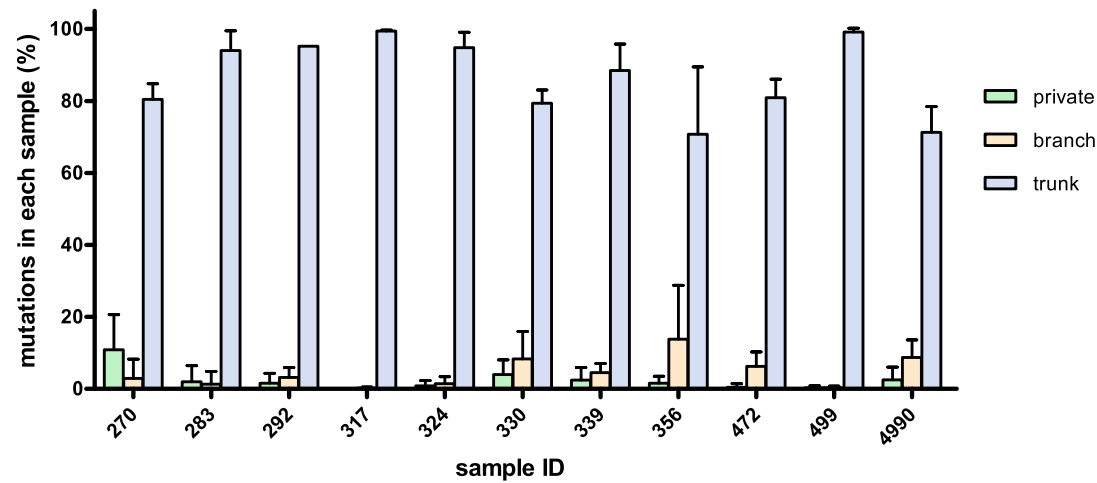

B

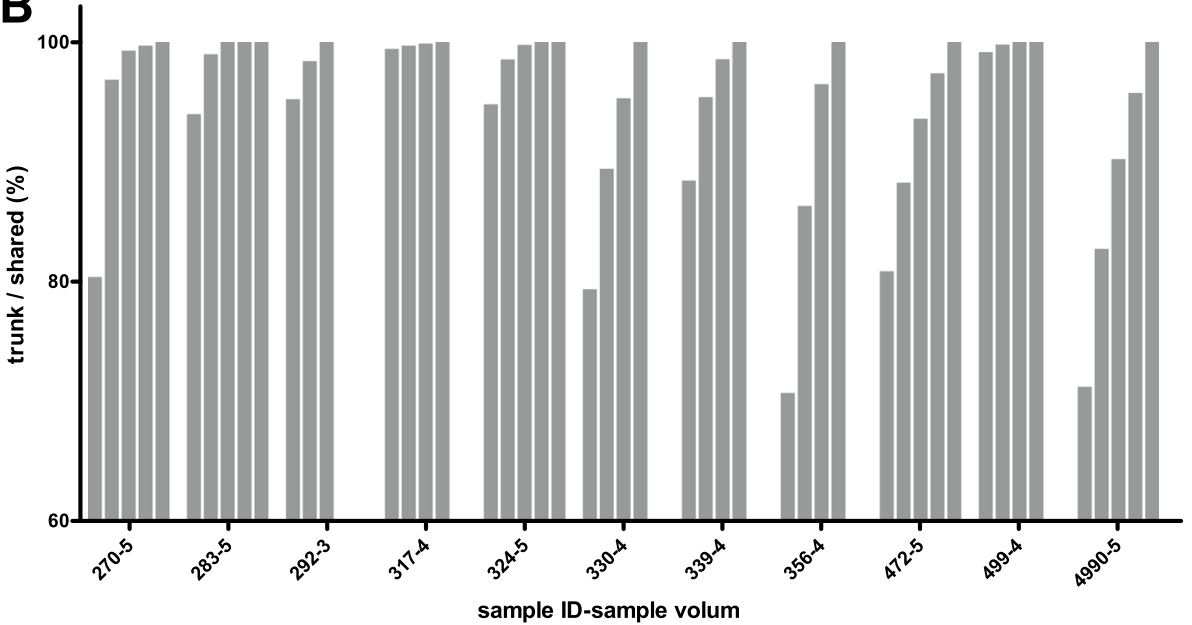

Fig. 3 Mutation proportions of lung adenocarcinomas across samples. a Distribution of exonic nonsynonymous SNVs in 11 tumors. The color bars indicate classification of mutations according to whether they are trunk (blue), branch (pink) or private (green) mutations. The $x$-axis shows the sample IDs of the 11 tumors. $\mathbf{b}$ The proportion of trunk mutations among the shared mutations of any set of samples, from one to maximum volume from each tumor. The $x$-axis indicates sample IDs with sample volumes. Detailed information is shown in Additional file 3: Fig. S3

mutations of any two samples and more than $90 \%$ of the shared mutations of any three samples were trunk mutations (Fig. 3b). This result is consistent with the results of the gastric carcinoma study.

\section{Trunk mutations tend to have higher variant allele frequencies}

We further analyzed the variant allele frequencies (VAFs) of the exonic nonsynonymous SNVs for the trunk and non-trunk mutations in gastric carcinoma. Most of the high-VAF mutations were located at the trunk mutation area (Fig. 4a). Generally, the VAFs of the trunk mutations (mean value $=0.30$ ) were significantly higher than those of the branch mutations (mean value $\left.=0.17, p<2.2 \times 10^{-11}\right)$ and private mutations $\left(\right.$ mean value $\left.=0.09, p<2.2 \times 10^{-16}\right)($ Fig. 4b). The VAFs in each sample also suggested that trunk mutations (mean value ranged from 0.23 to 0.33 ) occurred much more frequently than branch and private mutations (mean value ranged from 0.14 to 0.18 and 0.07 to 0.12 , respectively; Fig. 4c). However, the VAF of a certain mutation may vary in the same mutation type: the VAFs of trunk mutations varied from 0.12 to 0.52 , and those of branch and private mutations varied from 0.05 to 0.47 and 0.05 to 0.33 , respectively (Additional file 4). For example, the VAFs of trunk mutations in POM121L12 (mean VAF $=0.12)$ and RBM12 (mean VAF $=0.12)$ were less than the VAFs of private mutations in SHISA9 $(\mathrm{VAF}=0.33)$ and CCDC91 $(\mathrm{VAF}=0.24)$; this variation accounts for the observation that mutations with a high VAF are not always trunk mutations. Similarly, mutations with low VAF were not necessarily private mutations. Therefore, to identify trunk mutations, it is of great significance to utilize multiregional sequencing when considering mutations with high VAF as trunk mutations.

\section{Discussion and conclusions}

It is widely accepted that cancer is a process of microevolution [37, 38]. During the evolution of cancer, cells 


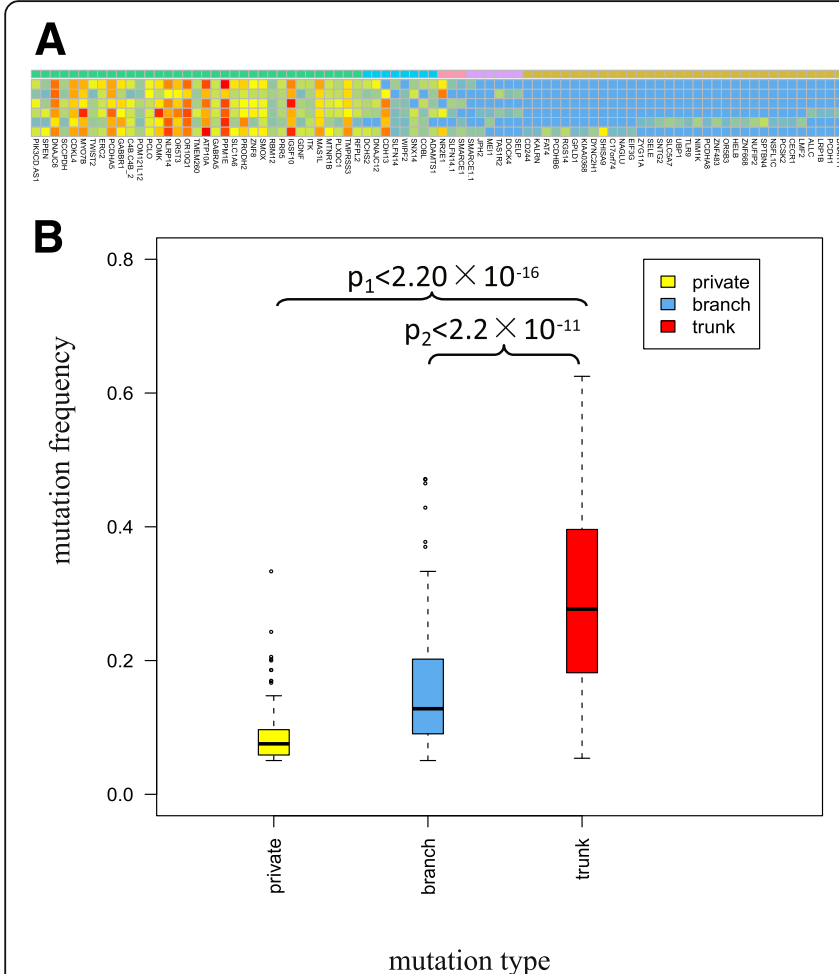

Fig. 4 Mutation frequency distribution in multiregional samples of gastric carcinoma according to mutation types. a Distribution of exonic nonsynonymous mutations in each region of sample with mutation frequency. $\mathbf{b}$ Mutation frequencies of all exonic nonsynonymous mutations, classified as private (yellow), branch (blue), and trunk (red) mutations. $\mathrm{p}_{1}$ shows a significant difference between the trunk and private mutations, and $\mathrm{p}_{2}$ shows a significant difference between the trunk and branch mutations. c Mutation frequency of three types of mutations in each sample. Gastric cancer $1\left(p_{1}=3.5 \times 10^{-6}, p_{2}=2.4 \times 10^{-4}\right)$, gastric cancer $2\left(p_{1}=1.6 \times 10^{-4}, p_{2}=3.8 \times 10^{-2}\right)$, gastric cancer $3\left(p_{1}=6.5 \times 10^{-8}, p_{2}=1.9 \times 10^{-5}\right)$, gastric cancer $4\left(p_{1}=5.4 \times 10^{-5}, p_{2}=2.7 \times 10^{-3}\right)$, gastric cancer $5\left(p_{1}=5.2 \times 10^{-8}, p_{2}=9.5 \times 10^{-2}\right)$, gastric cancer $6\left(p_{1}=8.6 \times 10^{-10}, p_{2}=9.3 \times 10^{-4}\right)$. The $p$ values were determined with a Wilcoxon test

experience a reiterative process of clonal expansion, diversification and selection [39]. Evidence of marked ITH shows the genetic diversity within cancer cells $[15,16,19,20]$ and suggests challenges to cancer precision medicine based on the mutational landscape portrayed by single-tumor biopsies [40]. Circulating tumor DNA (ctDNA) potentially reflects the biology of a cancer, which makes it a promising new biomarker for cancer diagnosis and prognosis [41, 42]. Searching for trunk mutations will contribute to expose potential targets in ctDNA for liquid biopsy. Moreover, with the development of targeted therapy and immunotherapy for cancer, tumor-specific mutated proteins, especially neoantigens, have attracted much attention due to their potential to be ideal targets for tumor immunotherapy $[8,10]$. It is clinically important to determine whether the tumor-specific mutated proteins are present in all cancer cells; their presence may determine the outcome of target therapy for cancer. Therefore, searching for trunk mutations, which occur in tumor-initiating cells and are present in all cancer cells, is of critical importance for cancer genomics.

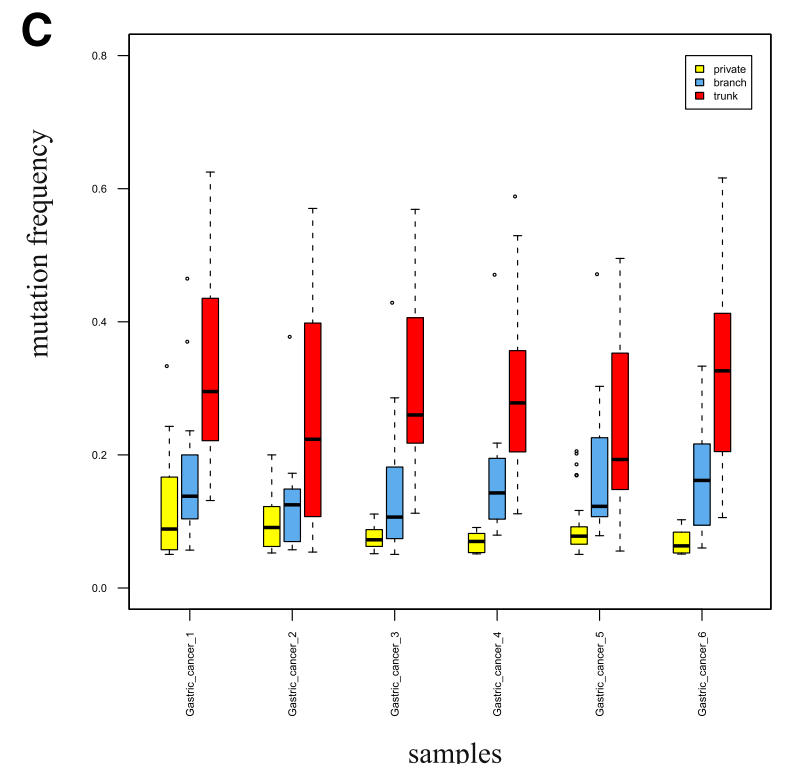


strategy for identifying trunk mutations for gastric carcinoma and lung adenocarcinoma, based on analyses of one gastric carcinoma and published data from 11 lung adenocarcinomas. However, ITH patterns might differ between cancer types. Studies involving more cancer types and larger cohorts will lead to a more complete understanding of the biological and therapeutic impacts of ITH.

\section{Additional files}

Additional file 1: List of simple somatic mutations across all six regions of the tumor, including SNVs and small INDELs. (XLSX $123 \mathrm{~kb}$ )

Additional file 2: Supplementary Table S1, S2, S3. (DOCX 23.5 kb)

Additional file 3: Supplementary Fig. S1-S3. (PDF $2620 \mathrm{~kb}$ )

Additional file 4: List of all 382 nonsynonymous SNVs from six samples originated from 160 exact mutation sites. (XLSX 21.7 kb)

Additional file 5: Function annotation tables of 160 mutated genes, including Interpro, Gene Ontology (GO), KEGG pathway, Online Mendelian Inheritance in Man (OMIM) according to the DAVID Bioinformatics Resources. (XLSX 57 kb)

\section{Abbreviations}

BWA: Burrows-Wheeler Aligner; GATK: Genome Analysis Toolkit; ICGC: International Cancer Genome Consortium; INDELs: Insertions/deletions; ITH: Intratumor heterogeneity; SNVs: Single nucleotide variants; VAFs: Variant allele frequencies; WES: Whole-exome sequencing

\section{Acknowledgements}

The authors thank the editor and reviewers for their critical comments to improve the manuscript. The authors also acknowledge the patient and his family for their understanding and support of this research project.

\section{Funding}

This work was supported by grants from the National Natural Science Foundation of China (31,501,021 and 81,430,081), the Zhejiang Provincial Natural Sciences Foundation of China (LY15C060001 and LY17H310001), and the Fundamental Research Funds for the Central Universities.

\section{Availability of data and materials}

The datasets generated and/or analyzed during the current study are available from the corresponding author upon reasonable request.

\section{Authors' contributions}

ZZ and SC designed and directed the research; ZZ, SW, YW and SC wrote the manuscript; ZZ, SW, JL, YS, CQ, JZ and ZC prepared and performed the experiments; and ZZ, SW, JZ, YW and XG performed the data analysis. All authors read and approved the final manuscript.

\section{Ethics approval and consent to participate}

The collection and use of the patient samples were approved by the Zhejiang Hospital of Traditional Chinese Medicine. Written informed consent was obtained from the participant. We confirmed that all methods were performed in accordance with the relevant guidelines (Approved Guidelines of the Clinical and Laboratory Standards Institute MM01-A3, MM13-A, and MM20-A, and the CARE Guidelines).

\section{Consent for publication}

Written informed consent was obtained from the patient for publication of this case report.

\section{Competing interests}

The authors declare that they have no competing interests.

\section{Publisher's Note}

Springer Nature remains neutral with regard to jurisdictional claims in published maps and institutional affiliations.

\section{Author details}

${ }^{1}$ College of Pharmaceutical Sciences, Zhejiang University, Zhejiang,

Hangzhou 310058, China. ${ }^{2} Z$ hejiang Hospital of Traditional Chinese Medicine, Zhejiang, Hangzhou 310058, China. 'Department of Biology and South Texas Center for Emerging Infectious Diseases, University of Texas at San Antonio, San Antonio, TX 78249, USA. ${ }^{\circ}$ Department of Genetics, Development and Cell Biology, lowa State University, Ames, IA 50011, USA. ${ }^{5}$ International Center for Precision Medicine, Zhejiang California International NanoSystems Institute, Zhejiang, Hangzhou 310058, China.

Received: 4 May 2017 Accepted: 13 July 2017

Published online: 17 July 2017

\section{References}

1. Stratton MR, Campbell PJ, Futreal PA. The cancer genome. Nature. 2009; 458(7239):719-24.

2. Hanahan D, Weinberg RA. Hallmarks of cancer: the next generation. Cell. 2011;144(5):646-74.

3. Vormehr M, Diken M, Boegel S, Kreiter S, Tureci O, Sahin U. Mutanome directed cancer immunotherapy. Curr Opin Immunol. 2016;39:14-22.

4. Wang Q, Chaerkady R, Wu JA, Hwang HJ, Papadopoulos N, Kopelovich L, et al. Mutant proteins as cancer-specific biomarkers. Proc Natl Acad Sci U S A. 2011;108(6):2444-9.

5. Heemskerk B, Kvistborg P, Schumacher TN. The cancer antigenome. EMBO J. 2013;32(2):194-203.

6. Tran E, Turcotte S, Gros A, Robbins PF, Lu YC, Dudley ME, et al. Cancer immunotherapy based on mutation-specific CD4+ T cells in a patient with epithelial cancer. Science. 2014;344(6184):641-5.

7. Lai J, Zhou Z, Tang XJ, Gao ZB, Zhou J, Chen SQ. A tumor-specific neoantigen caused by a Frameshift mutation in BAP1 is a potential personalized biomarker in malignant peritoneal Mesothelioma. Int J Mol Sci. 2016;17(5):739.

8. Schumacher TN, Schreiber RD. Neoantigens in cancer immunotherapy. Science. 2015;348(6230):69-74.

9. Rajasagi M, Shukla SA, Fritsch EF, Keskin DB, DeLuca D, Carmona E, et al. Systematic identification of personal tumor-specific neoantigens in chronic lymphocytic leukemia. Blood. 2014;124(3):453-62.

10. Desrichard A, Snyder A, Chan TA. Cancer Neoantigens and applications for immunotherapy. Clin Cancer Res. 2016;22(4):807-12.

11. Natrajan RC. Breast cancer heterogeneity: parallel evolution or conscious uncoupling? J Pathol. 2015;237(1):1-3.

12. Lawrence MS, Stojanov P, Polak P, Kryukov GV, Cibulskis K, Sivachenko A, et al. Mutational heterogeneity in cancer and the search for new cancerassociated genes. Nature. 2013:499(7457):214-8.

13. Kan Z, Jaiswal BS, Stinson J, Janakiraman V, Bhatt D, Stern HM, et al. Diverse somatic mutation patterns and pathway alterations in human cancers. Nature. 2010;466(7308):869-73.

14. Kandoth C, McLellan MD, Vandin F, Ye K, Niu B, Lu C, et al. Mutational landscape and significance across 12 major cancer types. Nature. 2013; 502(7471):333-9.

15. Zhang J, Fujimoto J, Zhang J, Wedge DC, Song X, Zhang J, et al. Intratumor heterogeneity in localized lung adenocarcinomas delineated by multiregion sequencing. Science. 2014;346(6206):256-9.

16. Patel AP, Tirosh I, Trombetta JJ, Shalek AK, Gillespie SM, Wakimoto H, et al Single-cell RNA-seq highlights intratumoral heterogeneity in primary glioblastoma. Science. 2014;344(6190):1396-401.

17. Navin N, Kendall J, Troge J, Andrews P, Rodgers L, Mclndoo J, et al. Tumour evolution inferred by single-cell sequencing. Nature. 2011;472(7341):90-4.

18. Ling S, Hu Z, Yang Z, Yang F, Li Y, Lin P, et al. Extremely high genetic diversity in a single tumor points to prevalence of non-Darwinian cell evolution. Proc Natl Acad Sci U S A. 2015;112(47):E6496-505.

19. Wang $Y$, Waters J, Leung ML, Unruh A, Roh W, Shi $X$, et al. Clonal evolution in breast cancer revealed by single nucleus genome sequencing. Nature. 2014:512(7513):155-60.

20. Gerlinger M, Rowan AJ, Horswell S, Larkin J, Endesfelder D, Gronroos E, et al. Intratumor heterogeneity and branched evolution revealed by multiregion sequencing. N Engl J Med. 2012;366(10):883-92. 
21. Gerlinger M, Horswell S, Larkin J, Rowan AJ, Salm MP, Varela I, et al. Genomic architecture and evolution of clear cell renal cell carcinomas defined by multiregion sequencing. Nat Genet. 2014;46(3):225-33.

22. Marusyk A, Almendro V, Polyak K. Intra-tumour heterogeneity: a looking glass for cancer? Nat Rev Cancer. 2012;12(5):323-34.

23. Hanahan D, Weinberg RA. The hallmarks of cancer. Cell. 2000;100(1):57-70.

24. McKenna A, Hanna M, Banks E, Sivachenko A, Cibulskis K, Kernytsky A, et al. The genome analysis toolkit: a MapReduce framework for analyzing nextgeneration DNA sequencing data. Genome Res. 2010;20(9):1297-303.

25. Cibulskis K, Lawrence MS, Carter SL, Sivachenko A, Jaffe D, Sougnez C, et al. Sensitive detection of somatic point mutations in impure and heterogeneous cancer samples. Nat Biotechnol. 2013;31(3):213-9.

26. Zhou Z, Lyu X, Wu J, Yang X, Wu S, Zhou J, et al. TSNAD: an integrated software for cancer somatic mutation and tumour-specific neoantigen detection. R Soc Open Sci. 2017;4(4):170050.

27. Li H, Durbin R. Fast and accurate long-read alignment with burrowswheeler transform. Bioinformatics. 2010;26(5):589-95.

28. Li H, Handsaker B, Wysoker A, Fennell T, Ruan J, Homer N, et al. The sequence alignment/map format and SAMtools. Bioinformatics. 2009;25(16):2078-9.

29. Wang K, Li M, Hakonarson H. ANNOVAR: functional annotation of genetic variants from high-throughput sequencing data. Nucleic Acids Res. 2010; 38(16):e164.

30. Huang da W, Sherman BT, Lempicki RA. Systematic and integrative analysis of large gene lists using DAVID bioinformatics resources. Nat Protoc. 2009; 4(1):44-57.

31. Kumar S, Stecher G, Tamura K. MEGA7: molecular evolutionary genetics analysis version 7.0 for bigger datasets. Mol Biol Evol. 2016;33(7):1870-4.

32. Futreal PA, Coin L, Marshall M, Down T, Hubbard T, Wooster R, et al. A census of human cancer genes. Nat Rev Cancer. 2004;4(3):177-83.

33. Forbes SA, Bindal N, Bamford S, Cole C, Kok CY, Beare D, et al. COSMIC: mining complete cancer genomes in the catalogue of somatic mutations in cancer. Nucleic Acids Res. 2011;39(Database issue):D945-50.

34. Oswald F, Kostezka U, Astrahantseff K, Bourteele S, Dillinger K, Zechner U, et al. SHARP is a novel component of the notch/RBP-Jkappa signalling pathway. EMBO J. 2002;21(20):5417-26.

35. Grasis JA, Browne CD, Tsoukas CD. Inducible T cell tyrosine kinase regulates actin-dependent cytoskeletal events induced by the $\mathrm{T}$ cell antigen receptor. J Immunol. 2003;170(8):3971-6.

36. Smith MJ, O'Sullivan J, Bhaskar SS, Hadfield KD, Poke G, Caird J, et al. Lossof-function mutations in SMARCE1 cause an inherited disorder of multiple spinal meningiomas. Nat Genet. 2013;45(3):295-8.

37. Cairns J. Mutation selection and the natural history of cancer. Nature. 1975; 255(5505):197-200.

38. Nowell PC. The clonal evolution of tumor cell populations. Science. 1976; 194(4260):23-8.

39. Greaves M, Maley CC. Clonal evolution in cancer. Nature. 2012;481(7381): 306-13.

40. McGranahan N, Swanton C. Biological and therapeutic impact of intratumor heterogeneity in cancer evolution. Cancer Cell. 2015;27(1):15-26.

41. Oxnard GR, Paweletz CP, Kuang Y, Mach SL, O'Connell A, Messineo MM et al. Noninvasive detection of response and resistance in EGFR-mutant lung cancer using quantitative next-generation genotyping of cell-free plasma DNA. Clin Cancer Res. 2014;20(6):1698-705.

42. Xi LQ, Pham THT, Payabyab EC, Sherry RM, Rosenberg SA, Raffeld M. Circulating tumor DNA as an early indicator of response to T-cell transfer immunotherapy in metastatic melanoma. Clin Cancer Res. 2016;22(22):5480-6.

\section{Submit your next manuscript to BioMed Central and we will help you at every step:}

- We accept pre-submission inquiries

- Our selector tool helps you to find the most relevant journal

- We provide round the clock customer support

- Convenient online submission

- Thorough peer review

- Inclusion in PubMed and all major indexing services

- Maximum visibility for your research

Submit your manuscript at www.biomedcentral.com/submit
Biomed Central 